Allergology today

\title{
Weizenallergie auf Haferbrei?
}

Welche fatalen Folgen unzureichende Deklarationen der Inhaltsstoffe von Lebensmitteln haben können, zeigt der Fall eines Säuglings mit einer Weizenallergie, den die Münchner Ernährungstherapeutin Dr. Imke Reese vorstellte:

Der Säugling, der seit seinem dritten Lebensmonat an atopischer Dermatitis litt, hatte im Alter von sechs Monaten nach dem Füttern eines neuen Breis ein QuinckeÖdem im Gesichtsbereich entwickelt. Verschiedene Gemüse sowie ein Milchbrei auf Reis-Mais-Basis wurden von dem Säugling problemlos vertragen. Im RAST-Verfahren (Radio-Allergo-Sorbent-Test) konnte der Verdacht auf eine Weizenallergie bestätigt werden. Der Mutter wurde empfohlen, bei der Ernährung nicht nur Weizen, sondern auch Hühnerei und Milch zu meiden. Da die Mutter noch stillte, verzichtete sie auch selbst auf diese Lebensmittel. Die eingeschränkte Ernährung führte zu einem hohen Leidensdruck, der die Mutter schließlich veranlasste, die Ernährungsberaterin aufzusuchen. Auf deren Rat hin nahm die Mutter wieder Ei und Milch in den eigenen Speisezettel auf, ohne dass allergische Reaktionen bei ihrem Kind auftraten oder dass sich dessen Hautzustand verschlechterte.

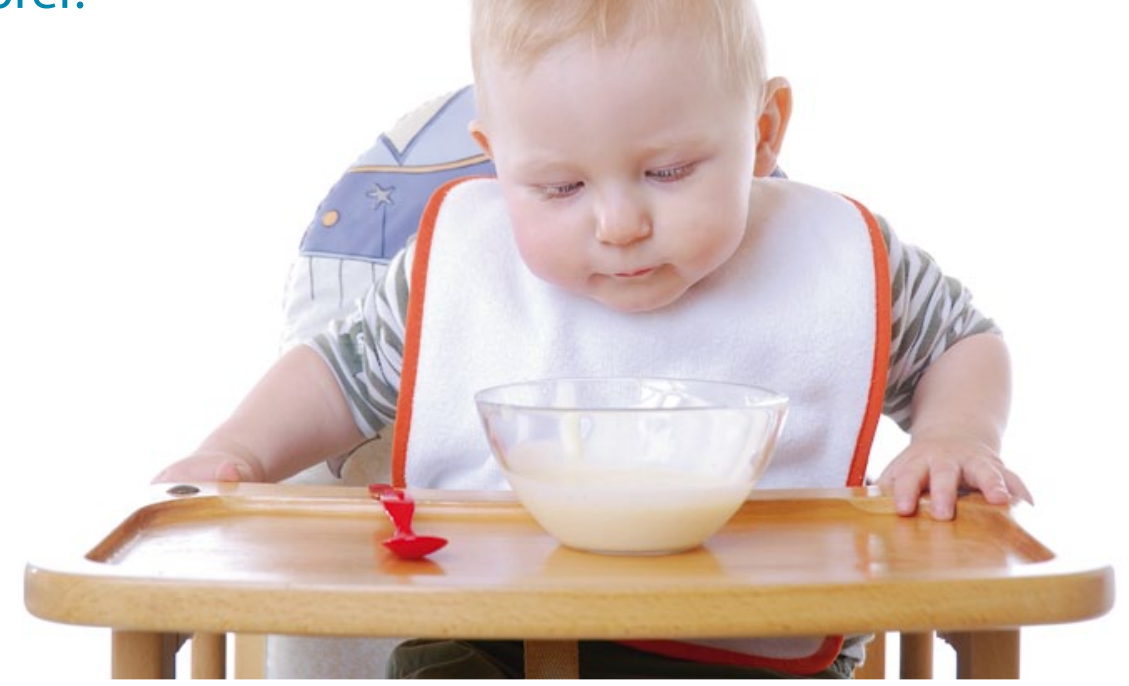

Vor Kontaminationen im Brei ist man nie gefeit.

Im Alter von neun Monaten kam es bei dem Säugling nach dem Verzehr von InstantHaferflocken zu einer anaphylaktischen Reaktion, obwohl Hafer vorher toleriert worden war. Daraufhin wurde eine Probe der Haferflocken an den Hersteller geschickt. Eine Analyse ergab eine Kontamination von mehr als 500 ppm Gluten, verursacht durch Dinkel oder Weizen. Das im Haferbrei „versteckte“ Getreide konnte da- mit als Auslöser einer erneuten heftigen allergischen Reaktion dingfest gemacht werden, so Reese. Der Hersteller habe zugesichert, künftig einen Hinweis auf eine mögliche Kontamination mit Dinkel oder Weizen auf der Verpackung des Haferbreis anzugeben.

Angelika Bauer-Delto

Reese I, Schmid L. Ungewöhnlicher Fall einer Weizenallergie

\section{Folsäure kann Urtikaria auslösen}

Über Allergien auf Vitamine ist bislang wenig bekannt. Umso ungewöhnlicher ist der Fall einer Folsäureallergie, den Dr. Michael Zirbs von der Klinik und Poliklinik für Dermatologie und Allergologie am Biederstein, München, präsentierte.

Bei Folsäure handelt es sich um die synthetische Form des Folat-B-Vitamins, das natürlicherweise vor allem in Blattgemüse, Vollkorn, Leber, Hefe, Milch und Eiern enthalten ist und für die Blutbildung eine wichtige Rolle spielt. Synthetische Folsäure wird häufig Multivitaminpräparaten und verschiedenen Lebensmitteln zugesetzt oder als Monopräparat verabreicht.

Der vorgestellte 81-jährige Patient entwickelte nach einer intramuskulären Injektion der Vitamine B1, B6 und B12 sowie von Folsäure eine akute Urtikaria mit Dyspnoe. Im Pricktest zeigte sich eine positive Reaktion auf Folsäure (siehe Abbildung), die anderen injizierten Vitamine blieben negativ. In der

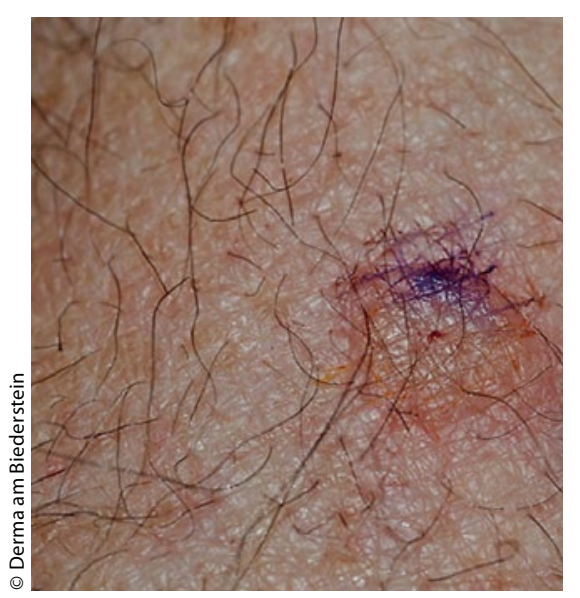

Hautreaktion beim Pricktest mit Folsäure
Epikutantestung wurden keine Hinweise auf eine Typ-IV-Sensibilisierung gefunden. Bei einer oralen, placebokontrollierten Provokationstestung kam es zu Allgemeinreaktionen auf Folsäure wie Flush, Schwindel und generalisiertem Pruritus, während die anderen Vitamine sowie Placebo vertragen wurden.

Dem Patienten wurde ein Allergiepass auf synthetische Folsäure ausgestellt. Ihm wurde angeraten, auf eine ausreichende Zufuhr natürlich vorkommender Folsäure in seiner Ernährung zu achten, die im Darm zu Tetrahydrofolsäure reduziert und vom Patienten gut toleriert wird. Angelika Bauer-Delto

Zirbs M, Radopoulou E, Cifuentes L, Pfab F, Ring J, Darsow U. Akute Urtikaria nach Folsäureinjektion 